\title{
Intracranial Intradural Vascular Injury during Endoscopic Endonasal Transsphenoidal Surgery: A Case Report and Literature Review
}

\author{
Mohammed Babgi ${ }^{10}$ \\ Saad Alsaleh ${ }^{2}$ \\ Yaser Babgi ${ }^{3}$ \\ ${ }^{1}$ Department of Surgery, Division of Neurosurgery, King Abdulaziz \\ University Hospital, Jeddah, Saudi Arabia \\ 2 Department of Otolaryngology, College of Medicine, King Saud \\ University, Riyadh, Saudi Arabia \\ ${ }^{3}$ Department of Surgery, College of Medicine, King Saud University, \\ Riyadh, Saudi Arabia
}

\author{
Saleh Baeesa ${ }^{1 \odot}$ Abdulrazag Ajlan ${ }^{3}$
}

J Neurol Surg Rep 2020;81:e52-e58.

\author{
Abstract \\ Keywords \\ - vascular injury \\ - endoscopic \\ endonasal approach \\ - endovascular \\ - intradural \\ - complication
}

Address for correspondence Abdulrazag Ajlan, MD, FRCSC, Department of Neurosurgery, King Khalid University Hospital, King Saud University, Riyadh, Saudi Arabia (e-mail: Dr_Ajlan79@Hotmail. com).Mailing Address: Department of Surgery, College of Medicine, King Saud University KKUH, Riyadh 11472, P.O.Box 7805 (37),

\section{Introduction}

Transsphenoidal surgery (TSS) has become widely accepted as the preferred approach not only for pituitary adenomas but also for other sellar or midline masses in the skull base. ${ }^{1-5}$ Complications of this approach are well documented, including cerebrospinal fluid (CSF) leak, diabetes insipidus, and infection. ${ }^{6-9}$ Among which are vascular complications that are rare and yet can lead to a fatal sequel, with an incidence of

received

February 14, 2020

accepted

June 25,2020
Background Transsphenoidal surgery (TSS) is a procedure for sellar or midline masses in the skull base. Among the reported complications are iatrogenic vascular injuries; that are rare, yet they carry devastating outcomes, with an incidence of injury between 0.34 and $2.6 \%$. The cavernous internal carotid artery is the most commonly injured. However, intradural arterial injuries are much less reported with challenging management. We report a rare incident of intradural arterial injury during TSS, and we compared our management to the summarized few cases reported in the literature Case Report We report a 43-year-old female who had a recurrent planum sphenoidal meningioma. She underwent trans-nasal transsphenoidal endoscopic resection that was complicated with intraoperative bleeding due to an injury to the anterior communicating artery that was challenging to control, resulted in a bilateral loss of flow in A1 segments of anterior cerebral artery and required endovascular management. The patient had a good recovery postoperatively without the typical picture of ACA syndrome.

Conclusion Intradural arterial injury is exceedingly rare in TSS, with no clear standard of care for the management. Collateral blood supply allows definitive management with minimal morbidity. Identifying the risk factors beforehand, as well as performing such cases in a well-resourced center, are crucial elements of safety. 
(embolus and stroke) in TSS are reported. ${ }^{10,13}$ In this paper, we report a case that had an injury to the ACA, resulting in bilateral ACA occlusion during endoscopic TSS for planum sphenoidale meningioma and will discuss these injuries and their management and outcomes.

\section{Case Report}

A 43-year-old female presented with a 1-year history of severe progressive decline in visual acuity. She underwent a left frontal craniotomy 8 years prior, for a subtotal resection of a large planum sphenoidal meningioma World Health Organization grade I ( - Fig. 1A-D). Neurological examination revealed cranial nerve functions within normal, apart from marked bilateral decreased visual acuity. Her motor, sensory, and coordination examination were unremarkable. The ophthalmologic assessment revealed significant impairment of visual acuity with only preserved light perception bilaterally; light reflexes and extraocular muscles were within normal.

Computed tomography (CT) of the brain showed left postsurgical frontal encephalomalacia with recurrent meningioma; there is marked underlying hyperostosis causing bilateral narrowing of optic canals (-Fig. 1E). Magnetic resonance imaging (MRI) scan demonstrated a recurrent enhancing mass measuring $5.4 \times 4 \mathrm{~cm}$ extending posteriorly into pituitary fossa and sphenoid and ethmoid sinuses inferiorly, with marked optic nerves and chiasm compression; there is marked increase in hyperostosis compared with her first MRI scan. The recurrent tumor enhanced homogeneously, as well as part of the hyperostosis, following intravenous contrast administration, and the ACA, A-comm complex was adherent to the tumor (-Fig. 1F-J).

The patient underwent endoscopic endonasal trans-sphenoidal surgery with navigation guidance. Surgery started

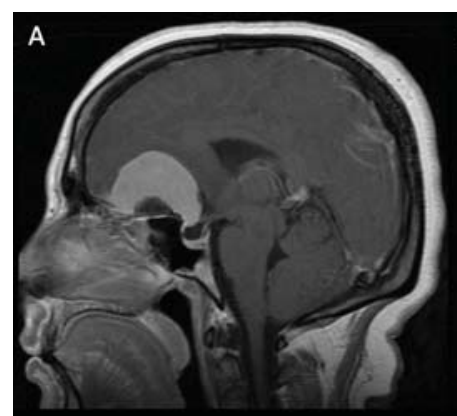

E
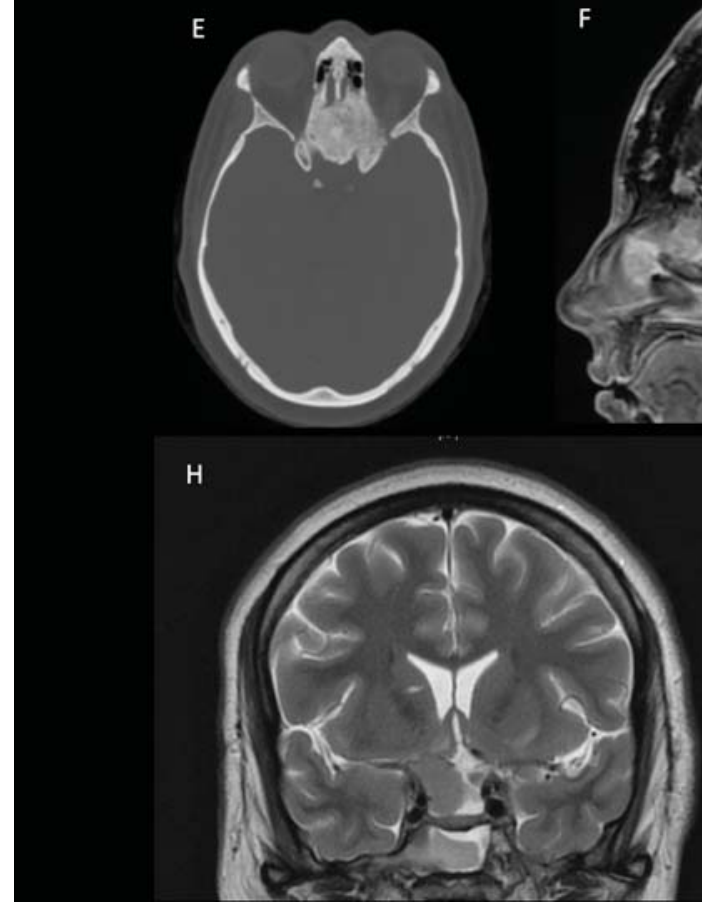

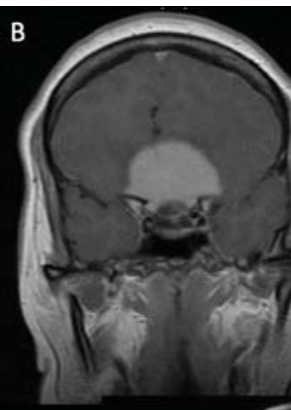

$\mathbf{F}$
H

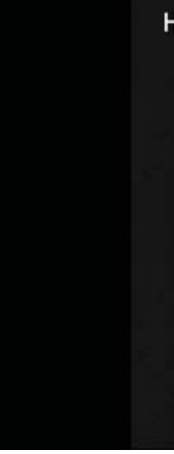

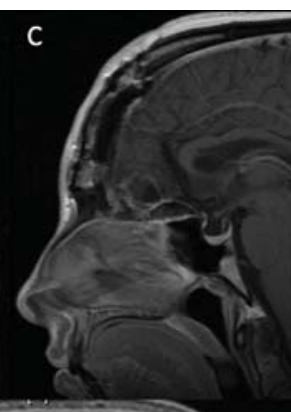

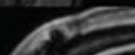

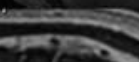

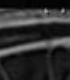

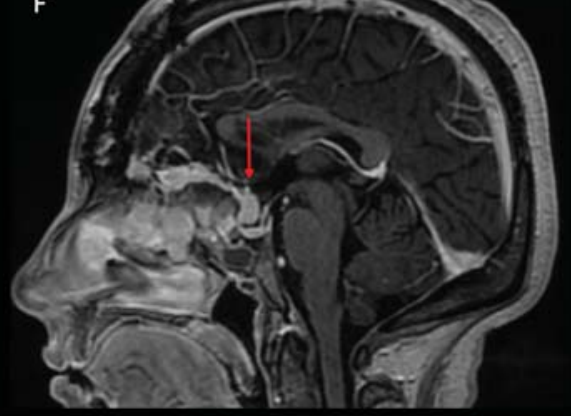

I

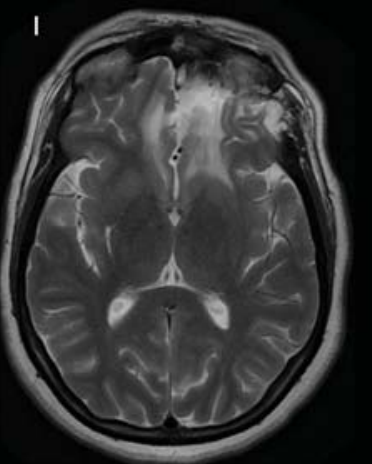

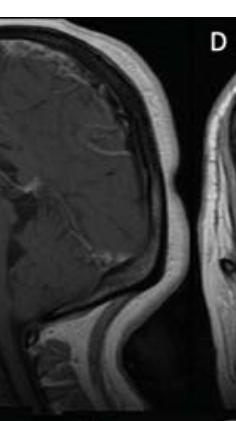

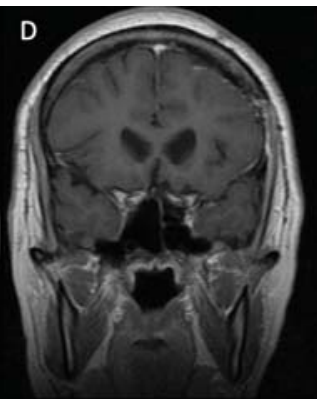

G
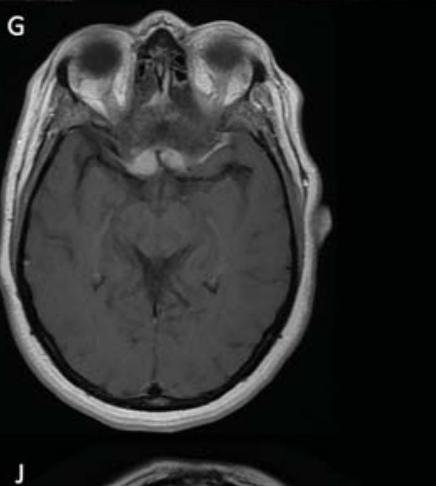

J

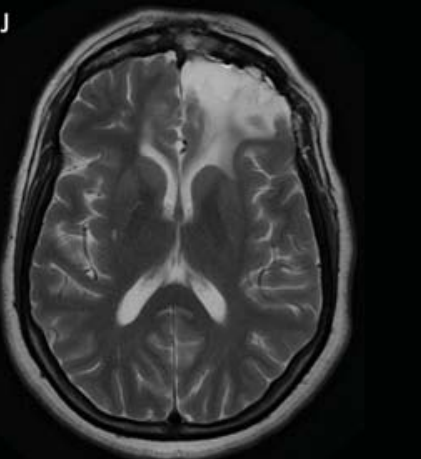

Fig. 1 (A, B) Axial and sagittal views of the brain MRI images (T1 with contrast) at first presentation 8 years ago. (C, D) Sagittal and coronal views of the brain MRI images (T1 with contrast) after first craniotomy performed 8 years ago. (E) Axial bone window computed tomography of the brain demonstrating hyperostosis of sphenoid and ethmoid bones, causing narrowing of both optic canals. (F, G) Sagittal and axial views of the brain MRI images (T1 with contrast) at current presentation demonstrating recurrent meningioma along planum sphenoidale with focal extension into the upper anterior portion of pituitary fossa. Note the proximity of the ACA-ACOM junction to the posterior border of the tumor (red arrow in sagittal view). Note the associated encephalomalacia, mainly involving the left frontal lobe from the previous craniotomy. (H, I, L) Coronal and axial views of the brain MRI images (T2) demonstrating the relation of the lesion to the right ICA-ACA arteries and the preexisting preoperative encephalomalacia, mainly involving the left frontal lobe. ACA, anterior cerebral artery; ACOM, anterior communicating artery; ICA, internal carotid artery; MRI, magnetic resonance imaging. 
with bilateral maxillary antrostomy, ethmoidectomy, sphenoidotomy, and nasoseptal flap elevation. There was part of the soft tumor in the ethmoid sinuses which was removed, bilateral optic nerve bone decompression was performed, and the hyperostotic skull base was drilled. The dura was then opened, and piecemeal removal of the tumor was performed. However, during dissection around the superior part of the tumor capsule, marked intracranial bleeding was encountered from what appeared to be from the A-comm artery. The bleeding source was challenging to visualize to apply an aneurysm clip and was controlled after packing with Gelfoam and Tachosil materials. The patient required the initiation of massive transfusion protocol due to significant blood loss ( $3 \mathrm{~L}$ ). Then surgery was concluded with skull base reconstruction in multiple layers, including the application of fat and fascia layers and nasoseptal flap followed by nasal packing. However, upon transferring the patient from the operative table, she started to bleed again from the nose. Then she was put back, and packing was removed, and skullbase corridor was reopened to identify the source that was discovered to be intradural coming from the A-comm artery area; the anatomy and extent of vascular injury was difficult to visualize, and was hardly stabilized after mounting pressure with hemostatic agents pulling the ACA-ACOM (anterior communicating) complex down against the planum with Penfield number 4 instrument.

After that, the neuro intervention team was emergently involved, and the patient was shifted to the angiogram suite with the packing and instrument in-place. The cerebral angiogram showed no flow in both A1 segments of ACA without extravasation or vasospasm; thus, bilateral A1 segments of ACAs were occluded to prevent recurrent hemorrhage and postoperative blood pressure (BP) augmentation using a coil on right and onyx on the left side (- Fig. $2 A-F$ ).

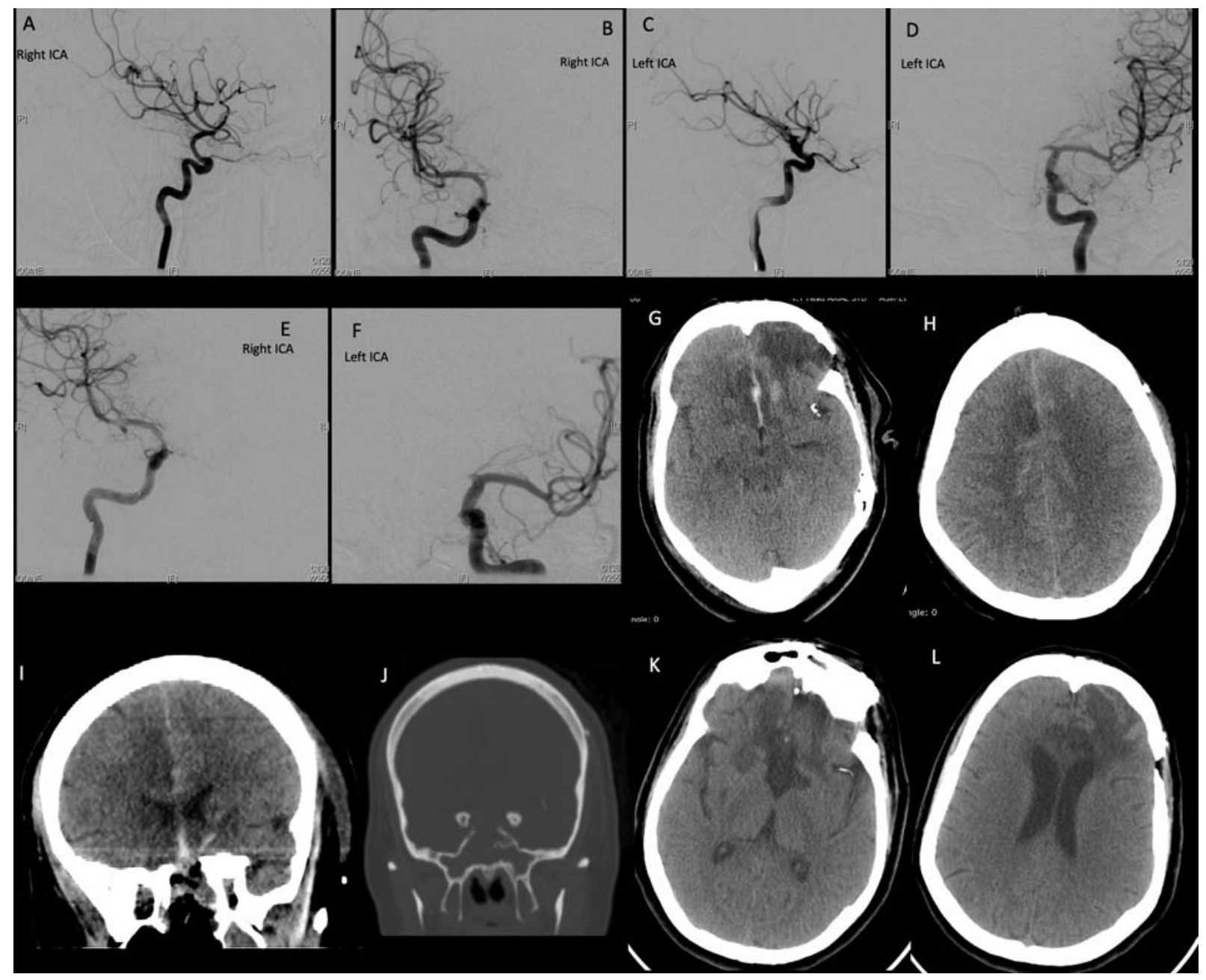

Fig. 2 (A, B) Lateral and anterior-posterior views of conventional angiography images of the right ICA that was performed immediately after the intraoperative incident of arterial injury that was temporarily stabilized, demonstrating the loss of flow in the first segment (A1) of right anterior cerebral artery before coiling. (C, D) Lateral and anterior-posterior views of conventional angiography images of the left ICA that also demonstrate similar description with loss of flow in A1. (E, F) Anterior-posterior views of conventional angiography images of the right and left ICA, demonstrating successful coiling on the right side and onyx embolization on the left side. $(\mathrm{G}, \mathrm{H}, \mathrm{I})$ Immediate postoperative axial and coronal views of the brain CT showing small hematoma at the surgical bed, subarachnoid hemorrhage, and small intraventricular extension, with only a tiny area of ischemic changes in the right paramedian frontal lobe. (J) Coronal bone window view of the brain CT obtained postoperatively demonstrating the decompression of the optic. (K, L) axial views of the brain CT obtained 4 days postoperatively (endoscopic endonasal surgery), showing the persistent stable right paramedian ischemic changes. CT, computed tomography; ICA, internal carotid artery. 
Afterward, the patient was brought back to the operative theater for redo construction of the skull base.

Immediate postoperative CT scan revealed a small hematoma at the surgical bed, subarachnoid hemorrhage, and small intraventricular extension, with only a tiny area of ischemic changes in the right paramedian frontal lobe ( - Fig. 2G-J). The patient was transferred to the intensive care unit (ICU) for monitoring, and BP augmentation above $160 \mathrm{~mm} \mathrm{Hg}$ systolic was ensued to induce collateral supply. She was extubated after 24 hours of surgery and remained hemodynamically stable. Her Glasgow coma score was 14/15 (due to confusion) and sustained right arm weakness of motor grade $1 / 5$, and bilateral lower limb weakness with a power of $2 / 5$ motor grade. CT scan revealed small areas of ischemic changes noted in left frontoparietal lobes and the anterior paramedian portion of the right frontal lobe (-Fig. $\mathbf{2 K}, \mathbf{L}$ ).

The patient was discharged from ICU after 2 weeks and required 6 months of daily care with physiotherapy and occupational therapy. She was then discharged to a rehabilitation center for further care. Upon discharge, she had normal lower limb power on the left side. However, on the right side, it was $3 / 5$, and she became able to walk with assistance. Regarding vision in comparison to preoperative assessment, ophthal- mological review reported no changes. MRI scan at 6-month follow-up demonstrated small residual lesion and stable findings regarding the ischemic changes (-Fig. $\mathbf{3}$ ).

\section{Discussion}

TSS is a relatively safe procedure compared with the open approach and has a less invasive approach and postoperative hospital-stay, with a mortality rate of less than $1 \% .{ }^{16}$ Complications rate in this surgery is estimated at around $6.3 \%$, accounting mainly for CSF leak and diabetes insipidus. ${ }^{6-8,10}$ Vascular complications during TSS are most feared and associated with limited visualization for its control intraoperatively with an incidence ranging between 0.34 and $2.6 \%{ }^{10-13}$ The majority of publications that concern vascular complications in TSS were accounted for ICA, as its injury is the most common in this approach. ${ }^{12}$ However, iatrogenic intradural arterial injuries are rare and reported cases in the literature are associated with variable morphology and management, with no clear standard of care (-Table 1). ${ }^{11,15-20}$ It is also worth noting that iatrogenic vascular complications in TSS are not necessarily to be observed intraoperatively, as in pseudoaneurysm or stenosis. ${ }^{19,21}$

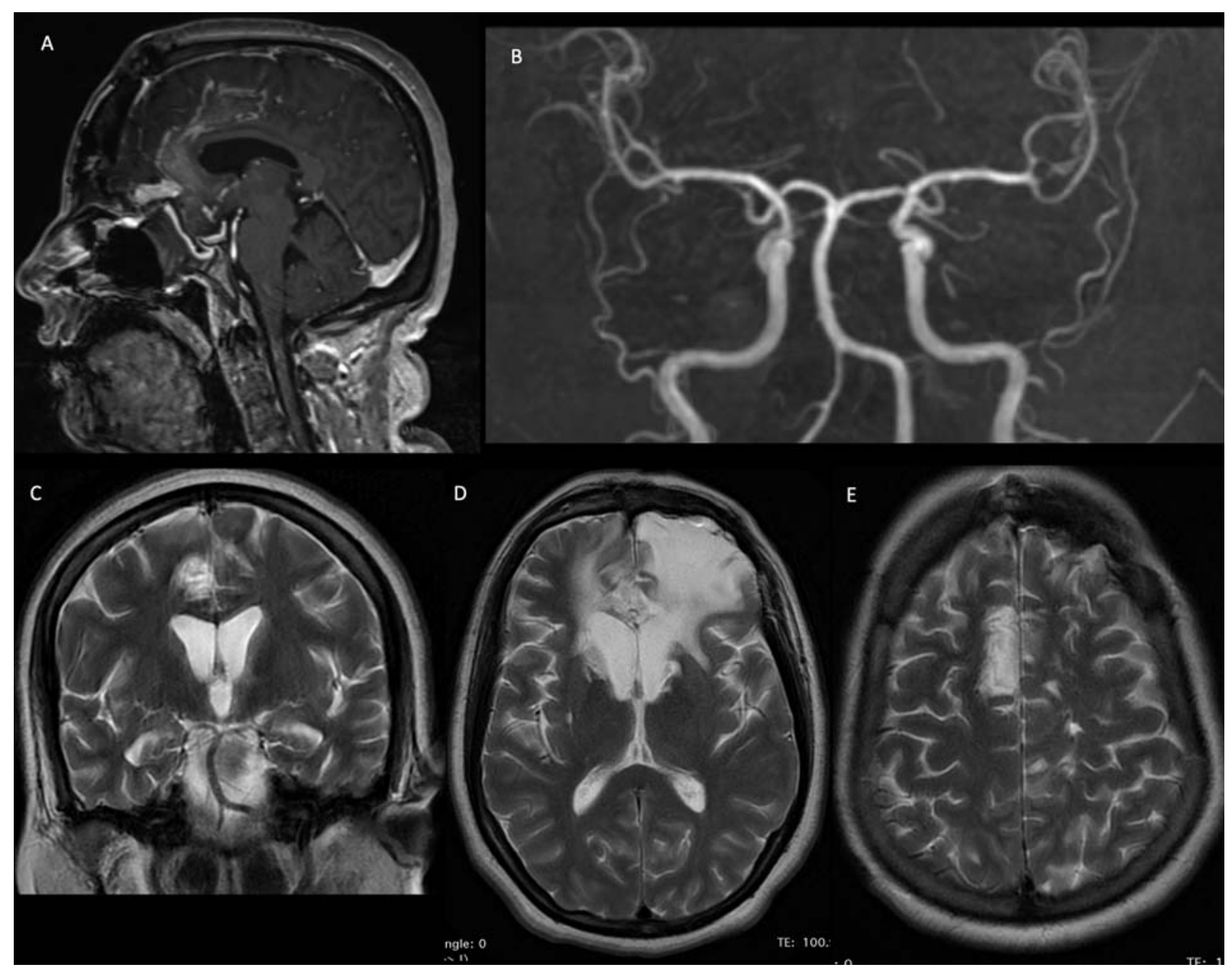

Fig. 3 (A) Sagittal MRI brain images (T1 with contrast) 6-month postoperatively (endoscopic endonasal surgery) demonstrating the small residual tumor and adequate reconstruction of the skull base floor. (B) Magnetic resonance angiography of the brain demonstrating persistent loss of flow in bilateral A1 segments. (C, D, E) Coronal and axial views of the brain MRI images (T2), demonstrating persistent right frontal lobe paramedian infarction with no interval ischemic changes. MRI, magnetic resonance imaging. 
e56 Intracranial Vascular Injury during Endoscopic Endonasal TSS Babi et al.

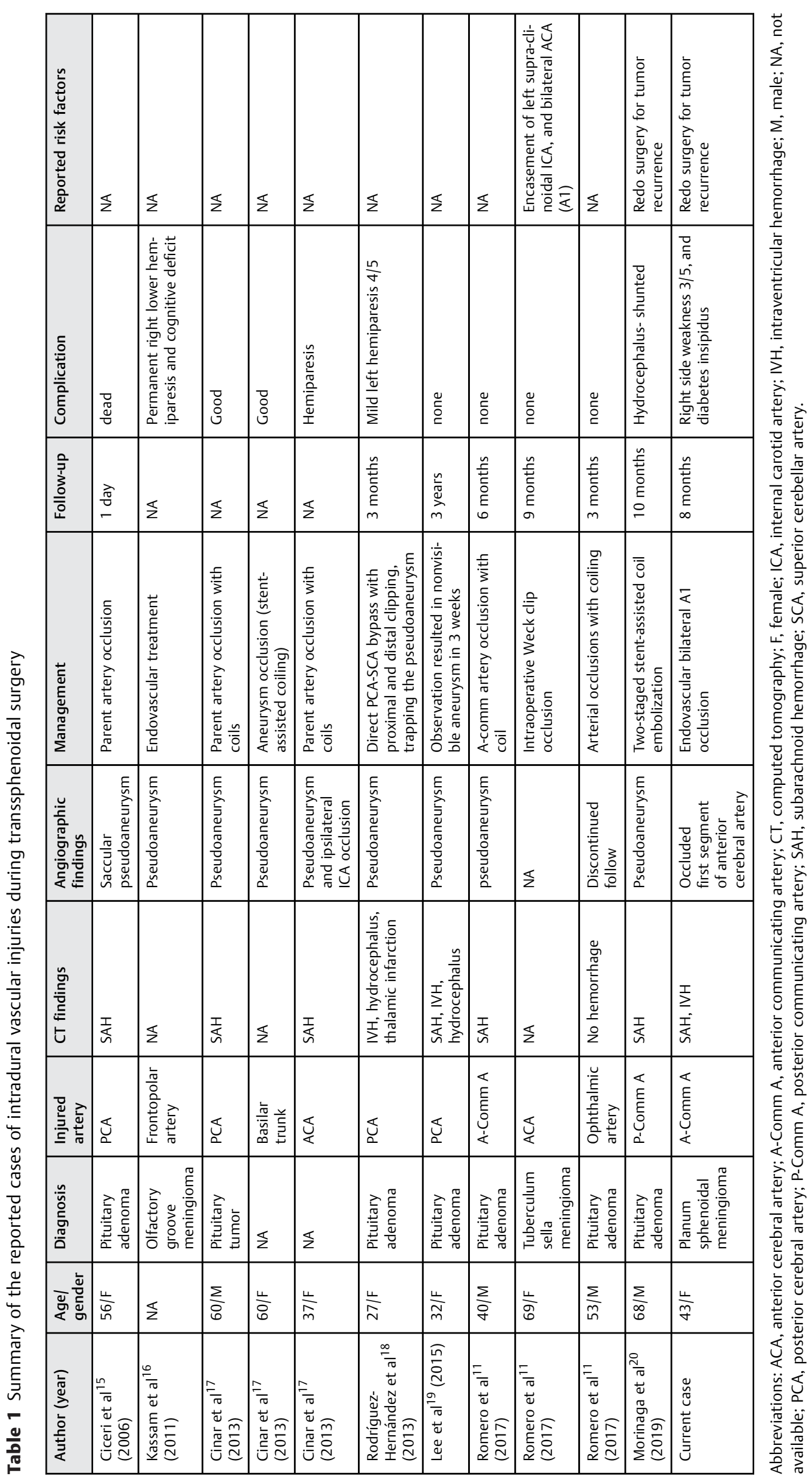

Journal of Neurological Surgery Reports Vol. 81 No. R3/2020 
We report a case that had an ACA complex injury in the form of ACOM artery injury that resulted in bilateral ACA occlusion during a redo TSS. Other intradural arterial injuries described include PCA, A-comm artery, P-comm artery, ophthalmic, and artery. Injuries are not necessarily recognized intraoperatively, and sometimes only associated with minor controllable bleeds. These cases are usually related to postoperative acute deficits or failure to wean off the intubation. ${ }^{18,19}$ Most of these injuries occur due to excessive manipulation or dissection that can avulse a perforator vessel adhered to the tumor and result in vascular wall compromise that is not well visualized in such a narrow operative corridor. ${ }^{18}$ In these cases, pseudoaneurysms are usually evident in CT angiography or a conventional angiogram (-Table $\mathbf{1}$ ).

In cases where intraoperative arterial injury with excessive bleeding is recognized, trials to control the bleeding intraoperatively using glue, cauterization, or muscle flap for tamponade are necessary to allow at least temporary control, allowing patients hemodynamic stability and subsequent transfer to the angiogram suite, as demonstrated in our case after quite challenging recurrent bleeding. There is a single case, however, reported by Romero et al that described intraoperative direct control of the parent artery (ACA) with weck clips occlusion after the failure of bipolar cauterization and muscle flap implantation. ${ }^{11}$

The rule of angiographic management after encountering intradural iatrogenic arterial injury is crucial, and the majority of reported cases ultimately required occlusion or sacrifice of the parent artery, especially when angiography shows adequate compensation from cross-circulation. ${ }^{11,15,17}$ Similar to most ICA injury cases during TSS, that was ultimately managed with endovascular parent artery occlusion. ${ }^{17,22}$ In one case reported by Romero et al, multiple trials to preserve the ACA had failed and the patient ultimately required occlusion of the parent artery. Furthermore, the patient experienced multiple dissecting aneurysms in the ICA. ${ }^{11}$ A recent case reported by Morinaga et al described a P-comm artery aneurysm after TSS, which was managed with coiling and subsequently followed by a second stage stenting because of incomplete obliteration of the aneurysm, and parent artery preservation was successful. ${ }^{20}$ Parent artery preservation is recently becoming possible in certain arterial injury cases first described in ICA injuries. ${ }^{23}$ RodriguezHernandez et al report another novel technique to preserve blood supply; he described microsurgical arterial bypass and pseudoaneurysm entrapment as an option in a case of iatrogenic PCA injury that resulted in a satisfactory outcome. ${ }^{18}$

Postoperatively, we targeted a systolic BP of above $160 \mathrm{~mm} \mathrm{Hg}$ to reduce the ischemic penumbra. Endovascular arterial obliteration allowed us to safely augment the BP in the ICU, which we believe significantly affected the outcome in our case by minimizing ischemic insults. This practice of $\mathrm{BP}$ augmentation is also used in acute ischemia patients and demonstrated positive results. Neurological improvement was observed in $33 \%$ of patients who had an ischemic stroke and managed with BP augmentation having a systolic BP of 130 to $180 \mathrm{~mm} \mathrm{Hg}$ as a threshold. Furthermore, stroke patients who are admitted with systolic BP either above or below the range mentioned above $(130-180 \mathrm{~mm} \mathrm{Hg})$ had the worst outcomes. ${ }^{24-26}$

Our patient had small bilateral areas of infarction in ACA territory more evident on the right side demonstrated in postoperative CT and did not develop the full picture of ACA syndrome contrary to what we expected as we did bilateral occlusion of the ACA. She improved over time with extensive rehabilitation until she became minimally dependent, which suggests that collateral blood supply could compensate ACA occlusion to a significant extent. Previously, reported ACA injuries during TSS were all unilateral, with our case being unique in its bilateral involvement. When arteries are adherent or encased by tumor tissue, the risk of injury increases, other predisposing factors for iatrogenic vascular injury also include less surgical experience, prior TSS, intraoperative tract deviation, and radiation therapy exposure. ${ }^{13,21,27}$

The learning points out of this case is to studying the images before the surgery to appreciate the proximity or involvement if the artery to the targeted tumor. If the tumor indeed was adherent to an artery and endoscopic approach would not be optimal to have sufficient exposure with optimal visualization, one should consider craniotomy over the endoscopic endonasal approach, especially in a redo surgery or cases that have risk factors predisposing arterial injury. We operated on this patient in a center with a high level of care and resources that allowed us to access the angiogram facility in excellent timing. However, it is worth noting that such rare complications if occurred in a center with lower resources that outcome would be remarkably different.

\section{Conclusion}

Intradural arterial injury during TSS surgery is an exceedingly rare entity, with no clear standard of care to manage such cases. Collateral blood supply allows definitive management of vascular injuries with minimal morbidity, a concept to which our case illustrated significant support. Postoperative BP augmentation, along with comprehensive rehabilitation, is an essential factor for recovery. Identifying the risk factors beforehand, as well as performing such cases in a well-resourced center, are crucial elements in the management of these cases.

\section{Conflict of Interest}

None declared.

\section{References}

1 Miller BA, Ioachimescu AG, Oyesiku NM. Contemporary indications for transsphenoidal pituitary surgery. World Neurosurg 2014;82(6, Suppl):S147-S151

2 Matsuo T, Kamada K, Izumo T, Nagata I. Indication and limitations of endoscopic extended transsphenoidal surgery for craniopharyngioma. Neurol Med Chir (Tokyo) 2014;54(12):974-982

3 Cappabianca P, Cavallo LM, Esposito F, De Divitiis O, Messina A, De Divitiis E. Extended endoscopic endonasal approach to the midline skull base: the evolving role of transsphenoidal surgery. Adv Tech Stand Neurosurg 2008;33:151-199

4 Cavallo LM, Frank G, Cappabianca P, et al. The endoscopic endonasal approach for the management of craniopharyngiomas: a series of 103 patients. J Neurosurg 2014;121(01):100-113 
5 Jho HD. Endoscopic transsphenoidal surgery. J Neurooncol 2001; 54(02):187-195

6 Wang F, Zhou T, Wei S, et al. Endoscopic endonasal transsphenoidal surgery of 1,166 pituitary adenomas. Surg Endosc 2015;29 (06):1270-1280

7 Berker M, Hazer DB, Yücel T, et al. Complications of endoscopic surgery of the pituitary adenomas: analysis of 570 patients and review of the literature. Pituitary 2012;15(03):288-300

8 Chivukula S, Koutourousiou M, Snyderman CH, Fernandez-Miranda JC, Gardner PA, Tyler-Kabara EC. Endoscopic endonasal skull base surgery in the pediatric population. J Neurosurg Pediatr 2013;11(03):227-241

9 Charalampaki P, Ayyad A, Kockro RA, Perneczky A. Surgical complications after endoscopic transsphenoidal pituitary surgery. J Clin Neurosci 2009;16(06):786-789

10 Turel MK, Tsermoulas G, Reddy D, Andrade-Barazarte H, Zadeh G, Gentili F. Endonasal endoscopic transsphenoidal excision of tuberculum sellae meningiomas: a systematic review. J Neurosurg Sci 2016;60(04):463-475

11 Romero A, Lal Gangadharan J, Bander E, Gobin Y, Anand V, Schwartz T. Managing arterial injury in endoscopic skull base surgery. Operative Neurosurgery. 2015;1;

12 Berker M, Aghayev K, Saatci I, Palaoğlu S, Önerci M. Overview of vascular complications of pituitary surgery with special emphasis on unexpected abnormality. Pituitary 2010;13(02):160-167

13 Laws ER Jr. Vascular complications of transsphenoidal surgery. Pituitary 1999;2(02):163-170

14 Yang H, Li SG, Xiang X, et al. Clinical classification and individualized design for the treatment of basicranial artery injuries. Medicine (Baltimore) 2019;98(11):e14732

15 Ciceri EF, Regna-Gladin C, Erbetta A, et al. Iatrogenic intracranial pseudoaneurysms: neuroradiological and therapeutical considerations, including endovascular options. Neurol Sci 2006;27(05): 317-322

16 Kassam AB, Prevedello DM, Carrau RL, et al. Endoscopic endonasal skull base surgery: analysis of complications in the authors' initial 800 patients. J Neurosurg 2011;114(06):1544-1568
17 Çinar C, Bozkaya H, Parildar M, Oran I. Endovascular management of vascular injury during transsphenoidal surgery. Interv Neuroradiol 2013;19(01):102-109

18 Rodríguez-Hernández A, Huang C, Lawton MT. Superior cerebellar artery-posterior cerebral artery bypass: in situ bypass for posterior cerebral artery revascularization. J Neurosurg 2013;118(05): 1053-1057

19 Lee CH, Chen SM, Lui TN. Posterior cerebral artery pseudoaneurysm, a rare complication of pituitary tumor transsphenoidal surgery: case report and literature review. World Neurosurg 2015;84(05):1493.e1-1493.e3

20 Morinaga Y, Nii K, Sakamoto K, Inoue R, Mitsutake T, Hanada H. Stentassisted coil embolization for a ruptured posterior communicating artery pseudoaneurysm after endoscopic transsphenoidal surgery for pituitary adenoma. World Neurosurg 2019;123:301-305

21 Raymond J, Hardy J, Czepko R, Roy D. Arterial injuries in transsphenoidal surgery for pituitary adenoma; the role of angiography and endovascular treatment. AJNR Am J Neuroradiol 1997;18 (04):655-665

22 Ghatge SB, Modi DB. Treatment of ruptured ICA during transsphenoidal surgery. Two different endovascular strategies in two cases. Interv Neuroradiol 2010;16(01):31-37

23 Kim BM, Jeon P, Kim DJ, Kim DI, Suh SH, Park KY. Jostent covered stent placement for emergency reconstruction of a ruptured internal carotid artery during or after transsphenoidal surgery. J Neurosurg 2015;122(05):1223-1228

24 Leonardi-Bee J, Bath PM, Phillips SJ, Sandercock PAIST Collaborative Group. Blood pressure and clinical outcomes in the International Stroke Trial. Stroke 2002;33(05):1315-1320

25 Vemmos KN, Tsivgoulis G, Spengos K, et al. U-shaped relationship between mortality and admission blood pressure in patients with acute stroke. J Intern Med 2004;255(02):257-265

26 Rordorf G, Cramer SC, Efird JT, Schwamm LH, Buonanno F, Koroshetz WJ. Pharmacological elevation of blood pressure in acute stroke. Clinical effects and safety. Stroke 1997;28(11):2133-2138

27 Oskouian RJ, Kelly DF, Laws ERJ Jr. Vascular injury and transsphenoidal surgery. Front Horm Res 2006;34:256-278 